\section{Incyte's big IDO}

\section{By Michael J. Haas, Senior Writer}

Researchers at Incyte Corp. have developed a class of compounds that target IDO, ${ }^{1}$ an immunoregulatory enzyme that is involved in a key cancer tolerance pathway but thus far has not been heavily pursued by biotech companies. Incyte hopes to have an IDO inhibitor in the clinic next year, which would put it behind NewLink Genetics Corp.'s 1-methyl-D-tryptophan, which is in Phase I testing for solid tumors. Incyte thinks its molecule could offer greater potency, but NewLink is already working on next-generation molecules that it says are more potent.

In 1998, a team at the Medical College of Georgia established an immunoregulatory role for indoleamine-pyrrole 2,3 dioxygenase 1 (IDO1; INDO; IDO) by showing that placental expression of the enzyme was an essential component of maternal tolerance toward the fetus. ${ }^{2}$ Since then, studies have found that IDO is expressed in both lymph nodes and tumors and that the molecule converts tryptophan to metabolites that shut down tumor-targeting T cells (see Figure 1, "Much IDO about cancer"). ${ }^{3}$

IDO overexpression is associated with poor prognosis in many cancers, including melanoma and colon, ovarian, breast and prostate cancer, as well as some hematological malignancies such as acute myelogenous leukemia (AML). The only disclosed IDO inhibitor in development is 1-methyl-D-tryptophan (1-MT; D-1MT).

The compound has moderate potency-30 $\mu \mathrm{M}$ - for IDO but does not inhibit the related liver enzyme tryptophan 2,3-dioxygenase (TDO2; TDO), which metabolizes dietary tryptophan to $\mathrm{N}$-formylL-kyurenine.

Given the dearth of potent, selective IDO inhibitors, the Incyte team began to search for a new scaffold targeting the enzyme.

Using high throughput screening and in vitro assays, the biotech team identified a hydroxyamidine compound with low micromolar activity against IDO. They synthesized and screened 13 hydroxyamidine analogs to identify a lead compound with low nanomolar activity against IDO and no activity against TDO.

In a mouse model of melanoma, the lead compound decreased blood plasma levels of kynurenine, a molecule that results from the metabolism of tryptophan by IDO and is thus a measure of IDO inhibition. The lead compound also reduced tumor growth compared with no treatment.
The hydroxyamidine inhibitors affected tumors regardless of whether they expressed IDO-indicating the compounds should be effective against non-IDO-expressing cancers via action on host IDO in the lymph nodes, according to team member Peggy Scherle, senior director of in vitro pharmacology at Incyte.

"But having said that, in the clinic we would first test cancers that overexpress IDO," she said.

Results were published in the Journal of Medicinal Chemistry. The Incyte team was led by Andrew Combs, executive director of chemistry.

The researchers have since identified another hydroxyamidine, INCB24360, as the company's clinical candidate.

\section{Undoing cancer's IDO}

Kenneth Lynn, EVP of business development at NewLink, said it was too early to comment on the therapeutic potential of Incyte's hydroxyamidine scaffold, including how the lead compound described in the $J M C$ article stacked up against D-1MT.

He did tell SciBX that "initial published data suggest that the lead compound may have limited oral bioavailability." Despite the in vitro assays suggesting Incyte's compound was more potent than D-1MT, Lynn said "its antitumor effect in murine models appears similar to D-1MT, which is orally bioavailable."

Scherle acknowledged that the lead compound described in the JMC article had limited oral bioavailability and an in vivo half-life of less than one hour. However, she noted that the clinical candidate, INCB24360, "has an oral bioavailability of approximately $50 \%$ in preclinical species and a half-life of $2-3$ hours in mice. Combined with its increased potency, INCB24360 does perform better than 1-MT in preclinical models."

Lynn said NewLink hopes to start Phase II studies of D-1MT by year end. "We're also engaged in discovery and development of potential second-generation IDO inhibitors," he said.

Both NewLink and Incyte think IDO inhibition has multiple selling points in cancer therapy.

First, IDO inhibition has synergistic effects with other cancer treatments. "Administration of $\mathrm{D}-1 \mathrm{MT}$ in combination with a variety of cytotoxic chemotherapeutic agents in several models of cancer has elicited regression of established tumors" without causing any observed increase in toxicity, Lynn said.

Incyte's Scherle agreed. "Preclinical results indicated that our clinical candidate, INCB24360, should be very effective in combination with chemotherapy or other immunotherapeutic strategies," she said.

Second, Lynn said that "by targeting a pathway that functions to a large extent outside of tumor cells, IDO inhibition should reduce the probability of the tumor developing resistance to the compound."

He added that a third advantage could be safety. "IDO knockout 


\section{TARGETS \& MECHANISMS}

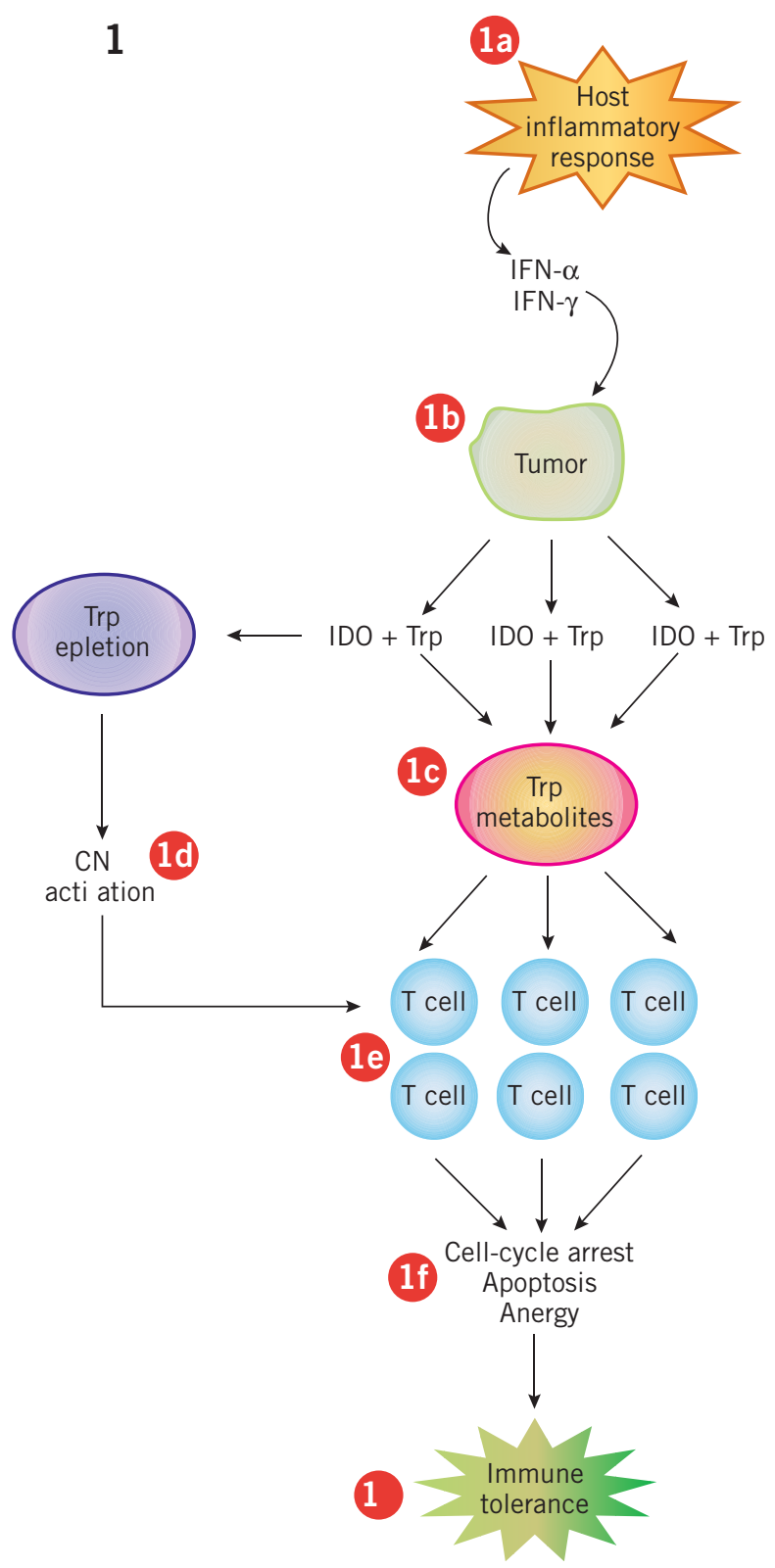

2

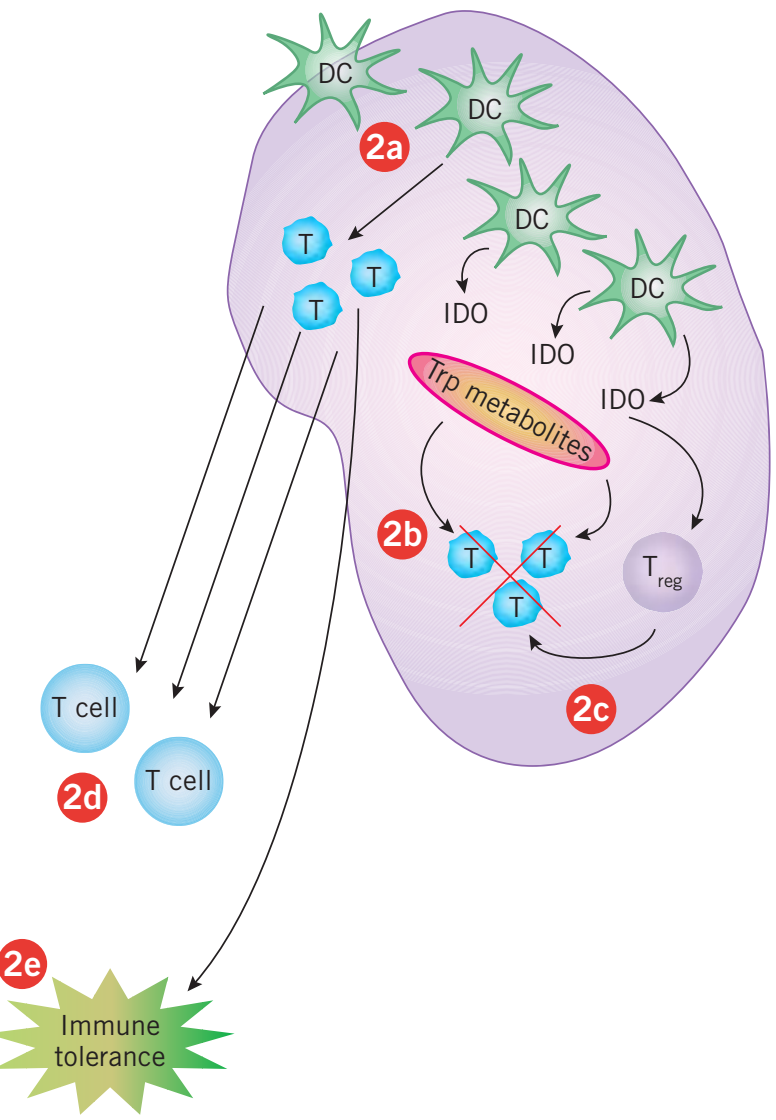

Figure 1. Much IDO about cancer. Expression of indoleamine-pyrrole 2,3 dioxygenase 1 (IDO1; INDO; IDO) can promote host immune tolerance in both the tumor microenvironment and the draining lymph nodes of the host. Thus, compounds inhibiting IDO could act against cancer in two different locales to decrease immune tolerance to certain tumors.

In the tumor microenvironment, the tumor induces a host inflammatory response that includes secretion of interferon- $\alpha$ (IFN- $\alpha$ ) and IFN- $\gamma$ [1a], which in turn can promote tumor secretion of IDO, which metabolizes tryptophan (Trp) [1 b]. This would lead to a local increase in Trp metabolites [1c] and local depletion of Trp; depleting Trp activates eukaryotic translation initiation factor $2 \alpha$ kinase 4 (EIF2AK4; GCN2) signaling [1d].

Trp metabolites and/or GCN2 signaling can cause tumor-targeting T cells [1e] to undergo cell-cycle arrest, apoptosis and/or anergy, all of which render them ineffective against the tumor [1f] and result in immune tolerance [1g]. Blocking IDO should prevent this cascade of events.

In the host lymph node, tumor antigen-presenting dendritic cells (DCs) activate tumor-targeting T cells [2a]. But IDO secreted by other types of DCs can increase levels of Trp metabolites and/or deplete Trp [2b]. IDO can also promote differentiation and activation of $\mathrm{T}_{\text {reg }}$ cells [2c], resulting in fewer tumor-targeting $\mathrm{T}$ cells [2d] and increased immune tolerance [2e]. Blocking IDO thus should result in the production of more tumor-targeting $T$ cells. 
mice are viable," he said. "This supports an expectation that inhibition of the IDO pathway might result in a favorable safety profile, which data so far have borne out."

Scherle said IDO inhibition induces none of the autoimmune problems that would indicate an undesirable effect on immune tolerance. "IDO is a brake on the immune system that doesn't play a role in normal autoimmune responses," she said.

\section{Prospects in tolerance}

Although both Incyte's and NewLink's programs are focused on cancer, mounting evidence suggests IDO also regulates immune tolerance mechanisms in indications such as asthma, autoimmune disease and transplant rejection. ${ }^{4}$

"Increased tryptophan degradation-which
"IDO is a brake on the immune system that doesn't play a role in normal autoimmune responses." -Peggy Scherle, Incyte Corp.
On the other hand, she cited another study indicating that IDOexpressing macrophages were important components of granulomas-nodules that wall off a pathogen the immune system cannot otherwise clear-suggesting that IDO inhibition could exacerbate diseases that are characterized by granulomas, such as Crohn's disease and tuberculosis (TB). ${ }^{7}$

Incyte expects to begin Phase I testing of INCB24360 to treat cancer in 2010, "but we need capital before starting clinical trials," said Combs.

Incyte, which had at least $\$ 175$ million in cash as of March 31, has seven compounds in the clinic to treat indications such as rheumatoid arthritis (RA), plaque psoriasis, multiple myeloma (MM), breast cancer, prostate cancer, type 2 diabetes and HIV infection.

is indicative of IDO upregulation-has been found in autoimmune syndromes, neurodegenerative disorders and in HIV, hepatitis C virus and Epstein-Barr virus infections," noted Reetta Huttunen, professor of internal medicine at Tampere University Hospital and the University of Tampere Medical School.

In May, Huttunen and colleagues reported that overexpression of IDO was associated with poor outcomes in sepsis. ${ }^{5}$

Noting that IDO inhibition has potential in chronic infectious diseases such as HIV and HCV, Lynn said NewLink intends to address $\mathrm{D}$-1MT's potential in these areas through partnering.

Scherle also said that infectious disease "is one other area of potential future interest" for IDO inhibitors. But both she and Robert Newton, VP of drug discovery biology at Incyte, said it was too early to say whether the company would pursue noncancer indications.

Huttunen also cautioned against assuming that IDO was as viable a target in infection as it may be in cancer. "IDO's precise role in different disease processes is unclear," she said.

On the one hand, Huttunen cited a study published in March showing that IDO blockade decreased proinflammatory responses and increased survival in an animal model of septic shocksuggesting IDO played a central role in that indication. ${ }^{6}$
The company will consider licensing or partnering INCB24360, according to spokesperson Pamela Murphy. Incyte has patented the findings reported in the $J M C$ paper.

Haas, M.J. SciBX 2(26); doi:10.1038/scibx.2009.1029

Published online July 9, 2009

\section{REFERENCES}

1. Yue, E. et al. J. Med. Chem.; published online June 9, 2009; doi:10.1021/jm900518f

Contact: Andrew P. Combs, Incyte Corp., Wilmington, Del. e-mail: acombs@incyte.com

2. Munn, D. et al. Science 281, 1191-1193 (1998)

3. Munn, D. \& Mellor, A. J. Clin. Invest. 117, 1147-1154 (2007)

4. Tan, P. \& Bharath, A. Expert Opin. Ther. Targets; published online June 17, 2009; doi:10.1517/14728220903018940

5. Huttunen, R. et al. Shock; published online May 18, 2009; doi:10.1097/SHK.0b013e3181ad3195

6. Jung, I. et al. J. Immunol. 182, 3146-3154 (2009)

7. Popov, A. et al. J. Clin. Invest. 116, 3160-3170 (2006)

COMPANIES AND INSTITUTIONS MENTIONED Incyte Corp. (NASDAQ:INCY), Wilmington, Del. Medical College of Georgia, Augusta, Ga. NewLink Genetics Corp., Ames, lowa Tampere University Hospital, Tampere, Finland University of Tampere Medical School, Tampere, Finland 